\title{
Trophoblast-Specific Conditional Atg7 Knockout Mice Develop Gestational Hypertension
}

\author{
Aiko Aoki, ${ }^{* \dagger \ddagger}$ Akitoshi Nakashima, ${ }^{*}$ Tae Kusabiraki, ${ }^{*}$ Yosuke Ono, ${ }^{*}$ Osamu Yoshino, ${ }^{*}$ Masanaga Muto, ${ }^{\dagger \S}$ Keiichi Kumasawa, \\ Tamotsu Yoshimori, ${ }^{\ddagger}$ Masahito Ikawa, ${ }^{\dagger}$ and Shigeru Saito*
}

From the Department of Obstetrics and Gynecology, ${ }^{*}$ University of Toyama, Toyama; and the Research Institute for Microbial Diseases, ${ }^{\dagger}$ the Department of Genetics, ${ }^{\ddagger}$ Graduate School of Medicine, the Graduate School of Pharmaceutical Science, ${ }^{\S}$ and the Department of Obstetrics and Gynecology, ${ }^{\uparrow}$ Osaka University, Suita, Japan

Accepted for publication

July 10, 2018.

Address correspondence to Shigeru Saito, M.D., Ph.D., 2630 Sugitani, Toyama 9300194, Japan. E-mail: s30saito@med.u-toyama.ac.jp.

\begin{abstract}
Hypertensive disorder of pregnancy (HDP) is a serious pregnancy complication that is life threatening to both the mother and fetus. Understanding HDP pathophysiology is important for developing medical treatments. This study demonstrates the involvement of autophagy deficiency in adverse maternal and fetal outcomes using trophoblast-specific autophagy related (Atg)7, an autophagy-related protein, knockout mice. Atg7 conditional knockout (cKO) placentas were significantly smaller than controls in the spongiotrophoblast layer but not the labyrinth layer, which significantly elevated blood pressure in dams. A marker of autophagy deficiency, sequestosome 1/p62, was accumulated in giant trophoblast cells and in the spongiotrophoblast layer, accompanying increased apoptosis. However, neither proteinuria in dams nor fetal growth restriction was observed. Regarding trophoblast function, the number of trophoblasts migrating into the maternal decidua was significantly reduced, and the wall/lumen ratio of the spiral arteries was significantly increased in CKO placentas, suggesting shallow trophoblast invasion and inadequate vascular remodeling. The relative expression of placental growth factor mRNA was significantly decreased in CKO placentas compared with the control, likely causing poor placentation; however, other factors were unchanged in CKO placentas. This is the first report of autophagy deficiency leading to impaired placentation complicated by maternal HDP attributable to trophoblast dysfunction, and it suggests that placental autophagy is required for normal placentation. (Am J Pathol 2018, 188: 2474-2486; https://doi.org/10.1016/j.ajpath.2018.07.021)
\end{abstract}

Hypertensive disorder of pregnancy (HDP), which occurs in $6 \%$ to $17 \%$ of pregnancies, is a serious obstetric complication characterized by hypertension after 20 weeks, and is a major cause of maternal and neonatal morbidity and mortality. ${ }^{1,2}$ The pathogenesis of HDP, especially preeclampsia (PE), has been explained by a two-stage model of PE, involving shallow invasion of trophoblasts into the maternal uterine wall as a first step in the early stage of placentation. ${ }^{1,2}$ The impairment of vascular remodeling may also be a reason for the shallow invasion of trophoblasts during the early pregnancy period. Subsequently, the first step leads to the second step, endothelial dysfunction, which is related to the secretion of several antiangiogenic factors, including a soluble form of the vascular endothelial growth factor (VEGF) receptor 1 (sFLT1) and soluble endoglin. ${ }^{3-5}$
The pathogenesis of PE has been studied from several angles, such as genetic and antiangiogenic factors, immune responses, antioxidants, and inflammatory cytokines. ${ }^{6-8}$ Regarding the pathologic association with autophagy in PE, it was previously reported that impairment of autophagy in extravillous trophoblasts (EVTs), which is necessary for normal placentation in the early pregnancy period, led to

Supported by the Japan Agency for Medical Research and Development (AMED) grant 16gk0110018h0001, a Kanzawa Medical Research Foundation grant, a Tamura Science and Technology Foundation grant, a Yamaguchi Endocrine Research Foundation grant, a First Bank of Toyama Scholarship Foundation grant, and Ministry of Education, Culture, Sports, Science and Technology, Japan, grants 15H04980, 16K11082, and $17 \mathrm{~K} 11221$.

Disclosures: None declared. 
shallow EVT invasion and poor vascular remodeling in vitro. ${ }^{9}$ Furthermore, sequestosome (SQSTM)1/p62 (SQSTM1), an autophagy-specific substrate, ${ }^{10,11}$ was accumulated in EVTs in placental bed biopsy samples, which contained deeply invaded EVTs in the myometrium, from women with PE. ${ }^{9}$ Several reports, however, proposed that autophagy was up-regulated in PE placentas. ${ }^{12-14}$ Thus, the role of autophagy in the pathophysiology of PE remains controversial. ${ }^{15,16}$ In addition, the precise function of autophagy in trophoblasts for normal placentation has yet to be elucidated.

Autophagy is a well-conserved protein degradation system among eukaryotes from yeast to mammals. Autophagy related $(A T G) 7$ is an autophagy-related gene encoding an E1-like enzyme that functions in the two ubiquitin-like conjugation systems essential for autophagosome biogenesis. ${ }^{17,18} \operatorname{Atg} 7^{-1-}$ pups exhibit significant fetal growth restriction and die earlier than wild-type pups as a result of lower amino acid concentrations in the serum during a neonatal starvation period. ${ }^{19}$ The knockout of another essential autophagy-related protein, Atg5, led to the same phenotypes as observed in $\operatorname{Atg} 7^{-1-}$ pups. ${ }^{20}$ Although fetal growth restriction is observed in autophagy-deficient fetuses, maternal preeclamptic symptoms, such as hypertension and proteinuria, have not been examined in autophagy-deficient dams. Furthermore, it is still unknown whether fetal growth restriction is attributable to autophagy deficiency in the fetuses and/or the placentas of the dams.

Herein, we established a placenta-specific Atg7 conditional knockout (cKO) using lentiviral vector (LV)mediated placenta-specific gene manipulation combined with the Cre/loxP system, ${ }^{21,22}$ and we evaluated maternal symptoms, placental structure, and fetal growth to clarify the biological significance of sole placental autophagy. The Atg7 cKO placentas, in which SQSTM1 was markedly accumulated in parietal trophoblast giant cells and the spongiotrophoblast layer, were significantly smaller than controls. The maternal blood pressure (BP) was significantly elevated, but proteinuria did not occur in dams bearing Atg 7 cKO placentas. Moreover, fetal growth was not restricted in cKO mice. Thus, autophagy deficiency in the placenta is involved in the poor placentation accompanying elevated maternal BP, suggesting its correlation with the pathophysiology of PE.

\section{Materials and Methods}

\section{Lentiviral Vectors}

The HIV-1-based self-inactivating LV-CAG-enhanced green fluorescent protein (EGFP) and the integrase-defective lentiviral vector (IDLV)-CAG-Cre were prepared, as previously reported. ${ }^{21,22}$ LV-EGFP was used to visualize control placentas, whereas IDLV-Cre was used to specifically delete ATG7 in trophoblast cell lineage cells. P24 gag antigen concentrations were measured to determine the virus concentration, as described previously. ${ }^{22}$

\section{Mice and Lentiviral Transduction}

Atg $7^{\text {flox/flox }}$ mice with a C57BL/6 (Japan SLC Inc., Shizuoka, Japan) background were crossbred at least three times with B6D2F1 (Japan SLC Inc.) mice, and female $A t g 7^{\text {flox/flox }}$ mice were obtained. They were superovulated using human chorionic gonadotropin 48 hours after i.p. injection of pregnant mare serum gonadotropin and mated with $\operatorname{Atg} 7^{\text {flox/flox }}$ male mice obtained by the same crossbreeding method. Two-cell-stage embryos were cultivated at $37^{\circ} \mathrm{C}$ in an incubator for 48 hours to obtain blastocysts. The zona pellucida of the blastocysts was removed by acid Tyrode's, and blastocysts were then transduced with LV-EGFP or IDLV-Cre at $1 \times 10^{3} \mathrm{ng} / \mathrm{mL}$ for 4 to 5 hours. They were transplanted into pseudopregnant ICR strain mice (Japan SLC Inc.) wild-type mice on embryonic day (ED) 2.5. For the measurement of BP and protein concentration in urine in dams, LV-EGFP- or IDLV-Cre-transduced blastocysts ( $n=20$ to 25 per pseudopregnant mouse) were transplanted into different pseudopregnant mice, respectively (Figure 1A). For the evaluation of placental and fetal body weights, equal numbers of LV-EGFP- or IDLV-Cre-transduced blastocysts (LV-EGFP/IDLV-Cre $=1: 1, n=20$ to 25 per pseudopregnant mouse) were transplanted into the same pseudopregnant mouse (Figure 1A). The birth rate is shown in Supplemental Table S1. Placental and fetal body weights were measured immediately after delivery by cesarean section on ED 19.5. Atg7 deficiency was confirmed by the presence of mutant alleles in placentas but not in fetuses (Figure 1B) using the following PCR primers ${ }^{19}$ : forward primer A, 5'TGGCTGCTACTTCTGCAATGATGT-3'; reverse primer B, $5^{\prime}$-AAGCCAAAGGAAACCAAGGGAGTG-3'; and reverse primer C, 5'-CAGGACAGAGACCATCAGCTCCAC-3'. These primers were used as indicated in Supplemental Figure S1. These Atg7-deficient placentas were defined as Atg7 cKO placentas. On the other hand, Atg $7^{\text {flox/flox }}$ blastocysts transduced with the EGFP-expressing LV were defined as EGFP placentas and did not possess the mutant allele.

\section{Measurement of Blood Pressure}

The tail-cuff method with BP98A (Softron, Tokyo, Japan) based on photoplethysmography was used to measure BP while the mice were warmed on a warming pad, as previously described. ${ }^{23}$ The mice were acclimated for 2 days to stay calm in the retainer. BP was measured at least five times when the values became stable. If data were not stable, $\mathrm{BP}$ was remeasured after a 15-minute interval. The data obtained from mothers carrying 5 to 10 viable fetuses were used in statistical analyses.

\section{Blood and Urinary Samples}

Blood samples were obtained from dam hearts after sacrificing. Urine samples were also collected on a plate from the 


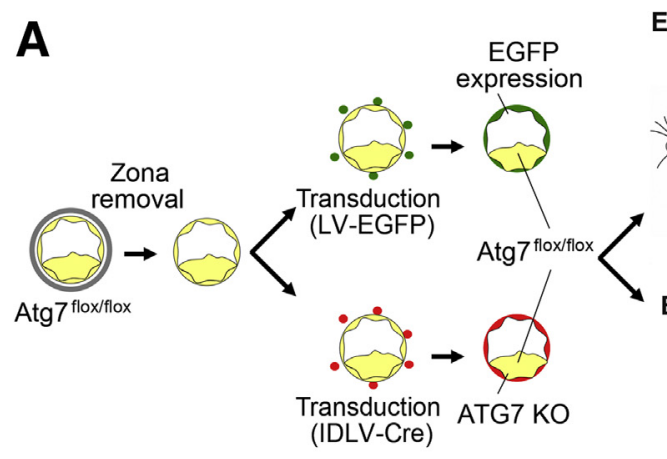

Exp.1: Analysis of blood pressure and protein concentration of urine

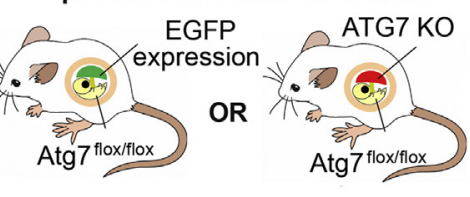

Exp.2: Analysis of placental and fetal weights ATG7 EGFP

KO expression
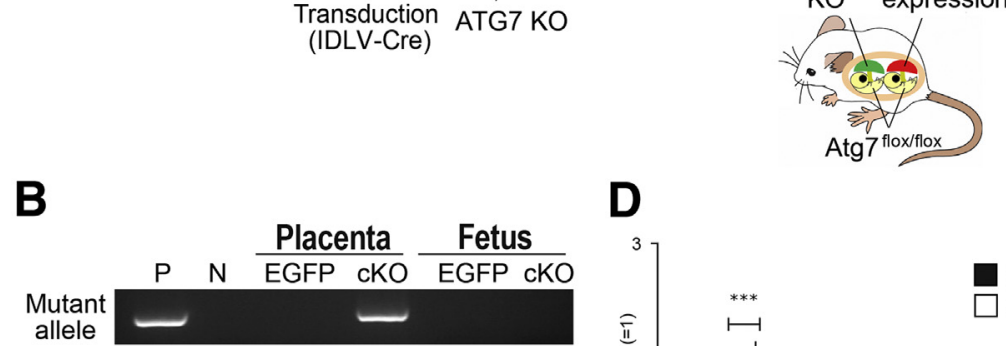

D

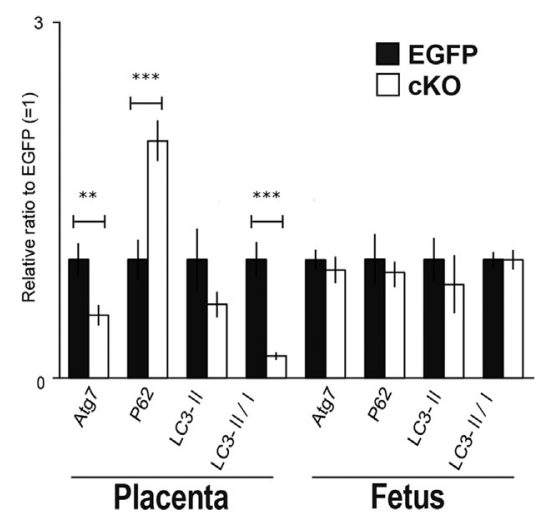

Figure 1 Lentiviral vector-mediated placentaspecific Atg7 knockout system in mice. A: $A \operatorname{tg} 7^{\text {flox/flox }}$ zygotes were obtained by mating $A t g 7^{\text {flox/flox }}$ females with $A t g 7^{\text {flox/flox }}$ males. After zona removal, the zygotes were divided into two groups, transduced with lentiviral vector (LV)-EGFP or integrase-defective LV (IDLV)-Cre. Then, they were transplanted into different pseudopregnant mice according to the LV type to evaluate blood pressure and proteinuria during pregnancy [experiment (Exp.) 1] or the same pseudopregnant mice after mixing the same number of LV-EGFP- or IDLV-Cre-transduced zygotes to evaluate placental and fetal body weights (Exp. 2). B: Genotyping of placentas and fetuses by PCR in the CKO system. All placentas and fetuses obtained were subjected to PCR to confirm the intended genotype. $C$ and D: Protein expression of Atg7, sequestosome 1 (SQSTM1; alias p62), and microtubule associated protein 1 light chain 3 beta (MAP1LC3B) in the placenta and fetus on embryonic day 19.5 by immunoblotting (C) and quantitative data (D). Loss of Atg7 expression is noted in cKO placentas but not in EGFP placentas. The fetuses from cKO placentas expressed Atg7. The star indicates a nonspecific band. ${ }^{* *} P<0.01,{ }^{* * *} P<0.001$. GAPDH, glyceraldehyde3-phosphate dehydrogenase; LC3, MAP1LC3B; $\mathrm{N}$, negative control; $\mathrm{P}$, positive control. pregnant dams to measure the protein concentration (Figure 2E). Blood samples were centrifuged to obtain serum samples. Concentrations of mouse sFLT1 and mouse VEGF were measured with enzyme-linked immunosorbent assay kits, according to the manufacturer's instructions (MVR100 and MMV00, respectively; R\&D Systems, Minneapolis, MN). Protein concentrations in urine were measured by Bradford analysis (5000006JA; Bio-Rad Laboratories Inc., Hercules, CA), according to the manufacturer's instructions.

\section{Immunohistochemistry}

Immunohistochemistry was performed as described previously. ${ }^{24}$ In brief, placentas were fixed in $4 \%$ paraformaldehyde overnight, dehydrated by ethanol and xylene, and then embedded in paraffin. The placenta/uterus blocks were sectioned vertically in the middle at a thickness of $8 \mu \mathrm{m}$. The sliced sections were deparaffinized in xylene, rehydrated in a graded series of alcohol, and then autoclaved in $10 \mathrm{mmol} / \mathrm{L}(\mathrm{pH}$ 6.0) citrate buffer for antigen retrieval at $121^{\circ} \mathrm{C}$ for 15 minutes. Endogenous peroxidase activity was blocked by $3 \%$ hydrogen peroxide in methanol for 15 minutes, and nonspecific binding was blocked by $5 \%$ normal goat serum. After extensive washing with phosphate-buffered saline, the sections were reacted with the rabbit polyclonal anti-SQSTM1 antibody (1:100; PM045; MBL, Osaka, Japan) at $4^{\circ} \mathrm{C}$ overnight. The sections were processed according to the manufacturer's instructions provided with the Vectastain kit (PK6200; Vector Laboratories, Burlingame, CA). For control sections, the primary antibody was replaced with control nonimmune rabbit IgG (sc-2027; Santa Cruz Biotechnology, Dallas, TX). Other primary antibodies used were as follows: anti-ATG7 (1:100; GTX32459; GeneTex Inc., Irvine, CA), anti-keratin$8(0.2 \mu \mathrm{g} / \mathrm{mL}$; AB-531826; Developmental Studies Hybridoma Bank, Iowa City, IA), anti-hypoxia-inducible factor- $1 \alpha$ (1:200; NB100-479; Novus Biologicals, Littleton, CO), and anti-8-hydroxy-2'-deoxyguanosine $(1 \mu \mathrm{g} / \mathrm{mL}$; MOG-020P; NIKKEN SEIL Co, Ltd, Shizuoka, Japan). Terminal deoxynucleotidyl transferase-mediated dUTP nick end labeling (TUNEL) staining was performed with the DeadEnd Colorimetric TUNEL system (G7360; Promega, Fitchburg, WI), according to the manufacturer's instructions.

\section{Histologic Analysis}

The summed area of parietal trophoblast giant cells, the spongiotrophoblast layer, and the labyrinth layer were referred to as 

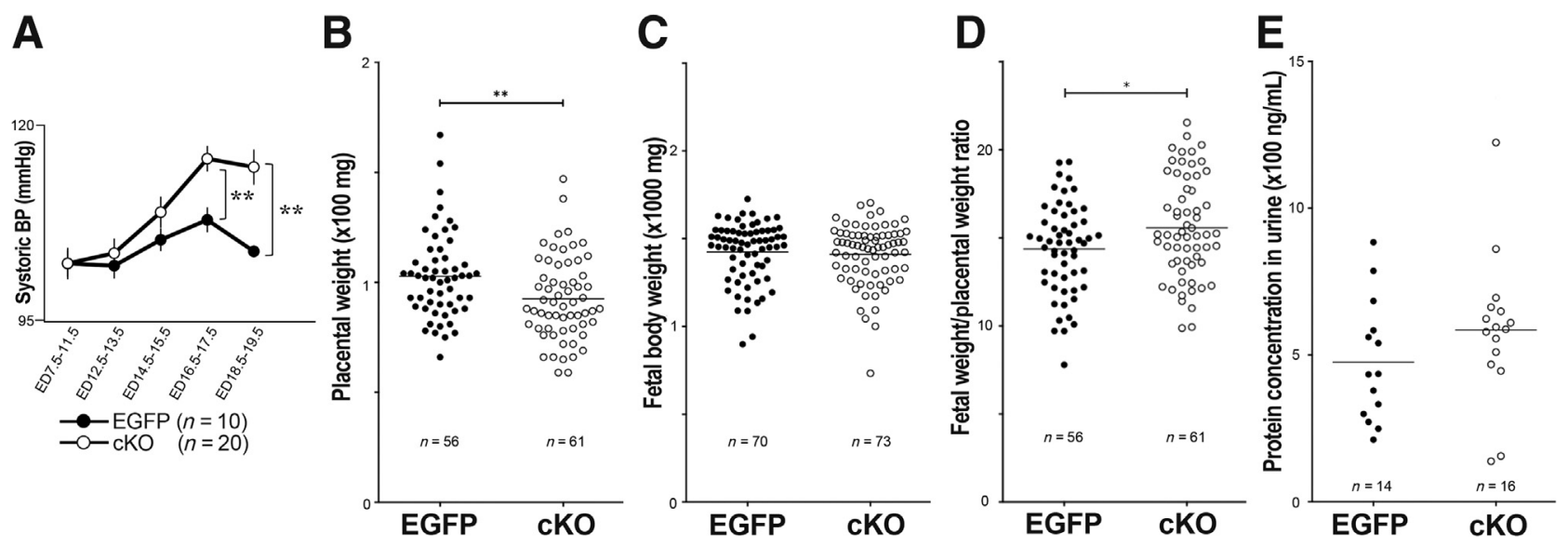

Figure 2 Hypertensive disorder of pregnancy phenotypes in dams with a placenta-specific autophagy-deficient placenta. A: Systolic blood pressure (BP) was measured in EGFP or CKO placenta-bearing dams on the indicated embryonic day (ED). B and C: Placental weight and fetal body weight were measured in EGFP and cKO groups on ED 19.5. The lines in the middle indicate the average weights in each group. D: The ratio of fetal weight/placental weight in both groups. The lines in the middle indicate the average of the ratio in each group. E: Protein concentration in urine, which was obtained during late pregnancy (ED 16.5 to 19.5), was measured in EGFP and CKO placenta-bearing dams. ${ }^{*} P<0.05,{ }^{*} * P<0.01$.

the total placental area. The area of the spongiotrophoblast and the labyrinth layers were each divided by the total placental area to calculate the relative ratio of each layer (Figure $3 \mathrm{~A}$ ). To count and calculate the ratio of keratin-8-positive cells, five fields were selected on the fetal side of the decidua in placentas. Horizontally sliced spiral arteries were selected to measure their wall/lumen ratios. TUNEL-positive signal intensity was measured in the middle part of each placental layer under $\times 200$ magnification, and the percentages of TUNEL-positive cells were calculated. ImageJ software version 1.41 (NIH, Bethesda, MD; https://imagej.nih.gov/ij) was used for histologic analyses.

\section{Western Blot Analysis}

Western blot analysis was performed as described previously. ${ }^{9,24}$ Placentas were homogenized in lysis buffer $(0.2 \%$ Triton in tris-buffered saline) with protease inhibitor cocktail (25955-11; Nacalai Tesque Inc., Kyoto, Japan) using a homogenizer (Mixer Mill MM 300; Retsch GmbH \& Co, Haan, Germany). Supernatants were collected after centrifugation at $16,890 \times g$ for 20 minutes at $4^{\circ} \mathrm{C}$. Each total protein extract $(50$ $\mu \mathrm{g})$ with $2 \%$ 2-mercaptomethanol (M3148; Sigma-Aldrich, St. Louis, MO) was boiled for 5 minutes, separated by SDS-PAGE, and transferred to a polyvinylidene difluoride membrane. The membrane was blocked for 60 minutes at room temperature with $1 \%$ skim milk in tris-buffered saline with $0.1 \%$ Tween 20 and incubated with each primary antibody shown below at $4{ }^{\circ} \mathrm{C}$ overnight. The following primary antibodies were used at the following concentrations: rabbit polyclonal anti-microtubule associated protein 1 light chain 3 beta (MAP1LC3B; 1:2000; PM036; MBL); rabbit polyclonal anti- SQSTM1 (1:1000; PM045; MBL); rabbit polyclonal anti-ATG7 (1:2000; 01322831; FUJIFILM Wako Pure Chemical Corp., Osaka, Japan); and mouse monoclonal anti-glyceraldehyde-3-phosphate dehydrogenase (1:1000; MAB374; Merck Millipore, Burlington, MA). The membranes were then treated with a secondary antibody diluted in tris-buffered saline with $0.1 \%$ Tween 20 and SuperSignal West Pico PLUS Chemiluminescent Substrate (34580; Thermo Fisher Scientific, Waltham, MA). The secondary antibody, horseradish peroxidase-linked anti-rabbit IgG (1:2000; 7074; Cell Signaling Technology, Danvers, MA) or horseradish peroxidase-linked anti-mouse IgG (1:4000; 7076; Cell Signaling Technology), was selected against the species in which the primary antibody was raised. The immunoreactive bands were detected using a chemiluminometer (MultiImager II; Mi-II 140CB; BioTools Inc., Gunma, Japan).

\section{Cell Culture}

Rcho- 1 cells were maintained and differentiated as previously described. ${ }^{25,26}$ The cells were cultured in RPMI 1640 medium (R8758; Sigma-Aldrich) containing $50 \mu \mathrm{mol} / \mathrm{L}$ 2-mercaptoethanol (M3148; Sigma-Aldrich), 1 mmol/L sodium pyruvate (1682049; FUJIFILM Wako Pure Chemical Corp.), and $20 \%$ fetal bovine serum for maintenance. NCTC-135 culture medium (N3262; Sigma-Aldrich), which contained 50 $\mu \mathrm{mol} / \mathrm{L}$ 2-mercaptoethanol, $1 \mathrm{mmol} / \mathrm{L}$ sodium pyruvate, and $1 \%$ horse serum, was used for the differentiation of Rcho- 1 cells.

\section{Chemicals}

Bafilomycin A1 (BAF; 88899-55-2; Cayman Chemical, Ann Arbor, MI), a lysosomal inhibitor, or Torin-1 (TRN1; 1222998-36-8; Cayman Chemical), a specific inhibitor of mammalian target of rapamycin, was used for in vitro experiments to manipulate autophagy in Rcho-1 cells.

\section{Invasion Assay}

The invasion assay was performed using a BD BioCoat Matrigel Invasion Chamber (354480; BD Biosciences, San 
A

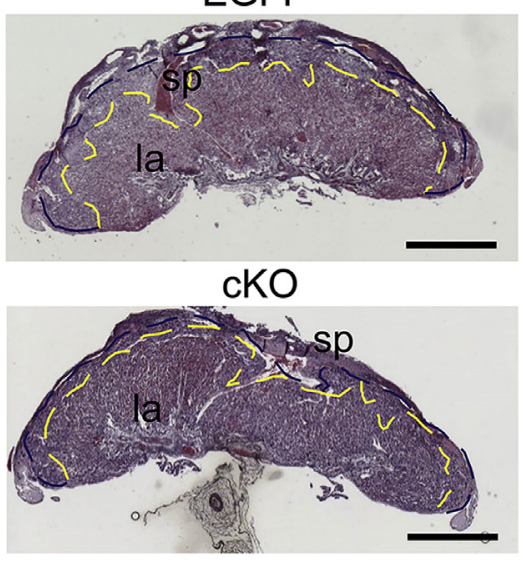

\section{B}

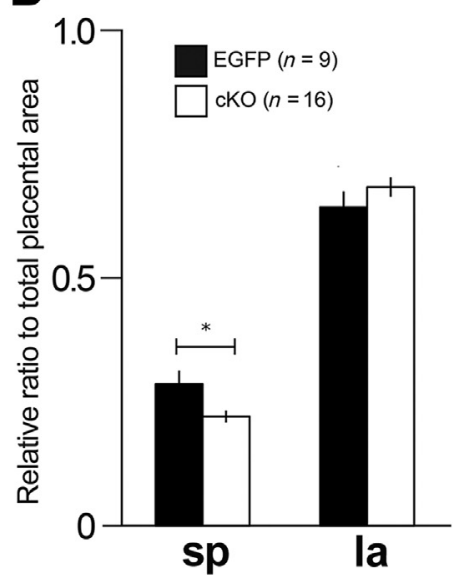

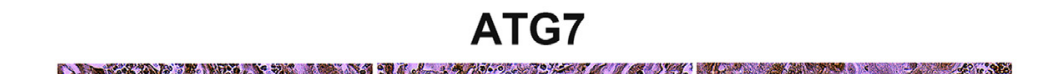

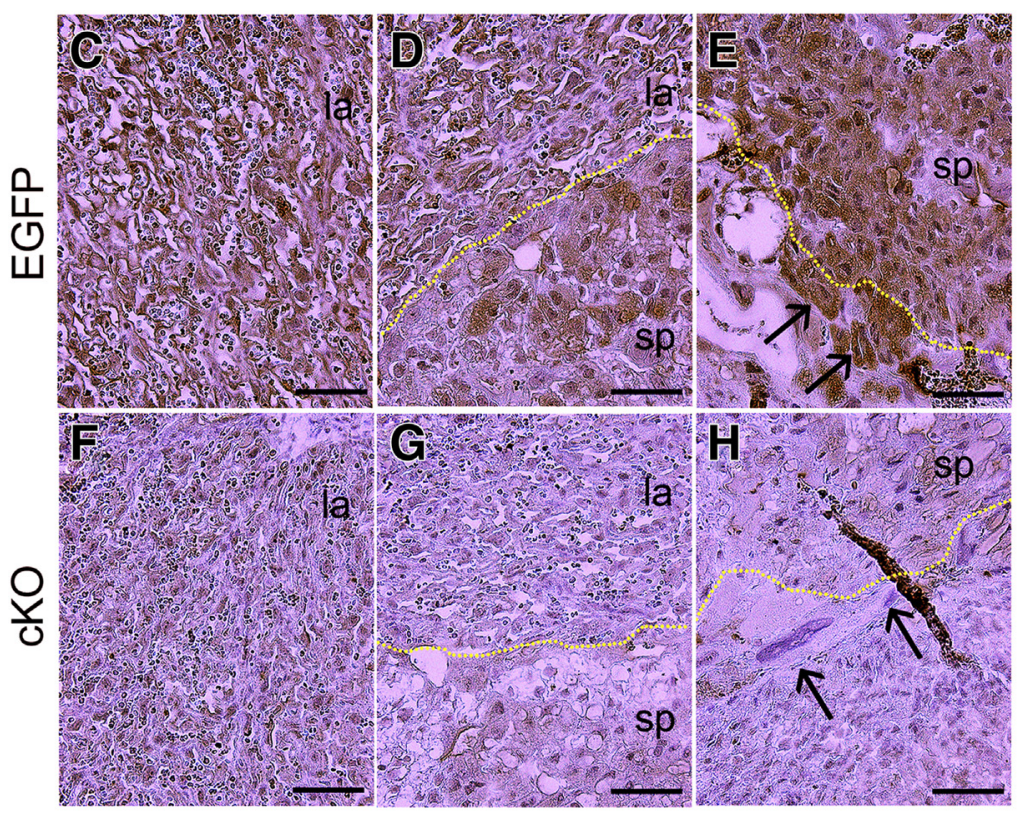

SQSTM1
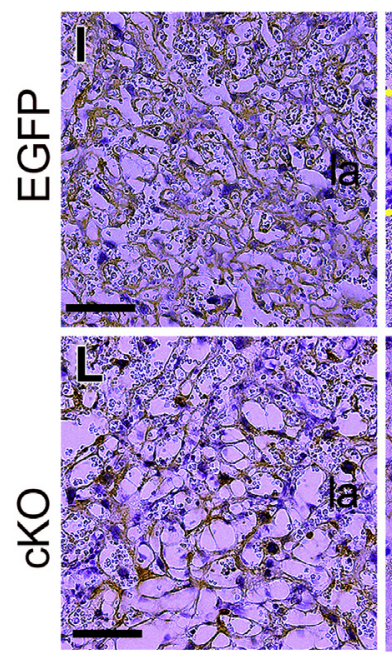
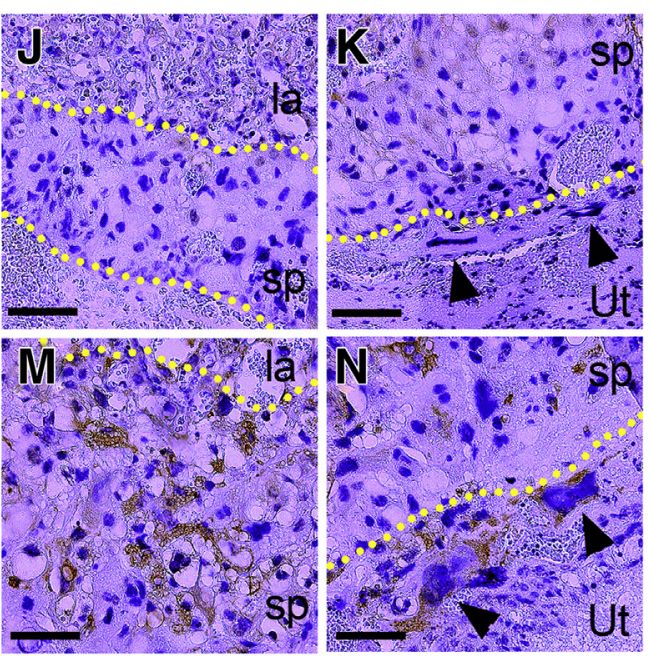

Figure 3 Histologic analysis of the EGFP and CKO placentas. A: Diagrams indicating murine placental structures comprising the labyrinth (la) and spongiotrophoblast (sp) layers. The parietal trophoblast giant cells make a thin layer between the spongiotrophoblast layer and maternal decidua. The labyrinth layers are outlined with a dashed yellow line, and spongiotrophoblast layers are between the dashed yellow lines and dashed blue lines. The upper side is the maternal side, and the lower side is the fetal side, in the presented diagrams. B: The graph shows the ratio of each area, spongiotrophoblasts or labyrinth layer, to the whole placenta area. Atg7 staining was performed for EGFP and CKO placentas. $\mathbf{C}-\mathbf{H}$ : Staining for Atg7 in placentas. Atg7 is expressed in the three placental layers, labyrinth layer (C), spongiotrophoblast layer (D), and parietal trophoblast giant cells (E) in the EGFP placentas, but not in the CKO placentas $(\mathbf{F}-\mathbf{H}) . \mathbf{D}, \mathbf{E}, \mathbf{G}$, and $\mathbf{H}$ : The dotted yellow lines indicate the borderline between labyrinth and spongiotrophoblast layers ( $\mathbf{D}$ and $\mathbf{G}$ ) and between spongiotrophoblast layer and decidua (E and $\mathbf{H})$. Arrows indicate parietal trophoblast giant cells. I-N: Staining for sequestosome 1 (SOSTM1) in placentas. I-K: In the EGFP placentas, SQSTM1 mainly localizes in labyrinth trophoblasts (I), but is not expressed in the spongiotrophoblast layer or parietal trophoblast giant cells ( $\mathbf{J}$ and $\mathbf{K})$. J: The top dotted yellow line indicates the borderline between labyrinth and spongiotrophoblast layers, and the bottom dotted yellow line indicates the borderline between spongiotrophoblast layer and decidua. $\mathbf{K}, \mathbf{M}$, and N: The dotted yellow lines indicate the borderline between labyrinth and spongiotrophoblast layers (M) and between spongiotrophoblast layer and decidua ( $\mathbf{K}$ and $\mathbf{N}$ ). Arrowheads indicate parietal trophoblast giant cells. Blue staining: nuclei by hematoxylin. L-N: In the cKO placentas, SQSTM1 staining is detected in the labyrinth layer (L), spongiotrophoblast layer (M), and parietal trophoblast giant cells $(\mathbf{N})$, which are proximal to the maternal decidua. ${ }^{*} P<0.05$. Scale bars: $1000 \mu \mathrm{m}$ (A); $50 \mu \mathrm{m}$ $(\mathbf{C}-\mathbf{N})$. Ut, uterine wall. 
Jose, CA), according to the manufacturer's instructions. ${ }^{27}$ In brief, cells were plated on the upper insert at $2 \times 10^{4}$ per well and incubated in the NCTC-135 differentiation medium with BAF ( 4 or $20 \mathrm{nmol} / \mathrm{L}$ ) or TRN1 ( $20 \mathrm{nmol} / \mathrm{L}$ ). These cells were incubated for 24 hours, and cells on the upper surface of the membranes were gently removed with a cotton swab. Then, the cells on the lower surfaces were fixed with $100 \%$ methanol (131-01826; FUJIFILM Wako Pure Chemical Corp.). Cell staining was performed with $0.05 \%$ Toluidine Blue Solution (206-14555; FUJIFILM Wako Pure Chemical Corp.). For each experiment, the number of cells was estimated by manual counting in 10 randomly chosen fields. Simultaneously, a cell proliferation assay was also performed under the same conditions as the invasion assay with the cell proliferation reagent WST-1 (MK400; Takara Bio Inc., Shiga, Japan), according to the manufacturer's instructions. The invaded cell index was calculated by dividing the number of invaded cells by the number of cultured cells in the presence of each chemical (Supplemental Figure S2A). These experiments were independently performed at least three times.

\section{Gelatin Zymography}

To measure the levels of secreted proteases, zymography assays were performed using a gelatin-zymography kit (AK47; Primary Cell Co Ltd, Hokkaido, Japan), according to the manufacturer's instructions. ${ }^{27}$ In brief, conditioned medium samples were collected from the wells in the invasion assays, and equal amounts of protein were applied and electrophoresed for 145 minutes. Enzyme reactions in the gel were then performed at $37^{\circ} \mathrm{C}$ for 48 hours to fully digest the substrate. The gels were stained with Coomassie Brilliant Blue, destained, and finally scanned by densitometry.

\section{Reverse Transcription and Quantitative Real-Time PCR Analysis}

This experiment was performed as described previously. ${ }^{28,29}$ In brief, total RNA was extracted from paraffin-embedded EGFP and cKO placentas using NucleoSpin total RNA formalin-fixed, paraffin-embedded kit (740982; Takara Bio Inc.), according to the manufacturer's instructions. Reverse transcription was performed using ReverTra Ace qPCR RT Master Mix with genomic DNA remover (FSQ-301; Toyobo Life Science, Osaka, Japan). Approximately 0.5 to $1 \mu \mathrm{g}$ of total RNA was reverse transcribed in a $20-\mu \mathrm{L}$ volume. For the quantification of mRNA levels, real-time PCR was performed using the Mx3000P Real-Time PCR system (Agilent Technologies, Santa Clara, CA). To evaluate the quality of mRNA obtained from the paraffin-embedded tissues for quantitative RT-PCR, the significant reduction in $\operatorname{Atg} 7 \mathrm{mRNA}$ in cKO placentas was first confirmed. Subsequently, the following PCR primers were used to determine placental and fetal genotypes in the cKO system. The primer sequences were as follows: Atg7 (forward primer A, 5'-TGGCTGCTACTTCTGCAATGATGT-3'; reverse primer B, 5'-
AAGCCAAAGGAAACCAAGGGAGTG-3'; reverse primer C, 5'-CAGGACAGAGACCATCAGCTCCAC-3'); placental growth factor $(P g f)$ (forward: $5^{\prime}$-TGTGTCCTTCTGAGTCGCTG-3' and reverse $5^{\prime}$-TCTGCCTTTGTCGTCTCCAG$\left.3^{\prime}\right)^{20}$; catechol-O-methyltransferase (Comt) (forward: $5^{\prime}-\mathrm{AG}-$ ACCGCTACCTTCCAGACA-3' and reverse: $5^{\prime}$-CTCATCAGGCTGAGTGGTCA-3'); and Eng (forward: 5'-CGATAGCAGCACTGGATGAC- $3^{\prime}$ and reverse $5^{\prime}$-AGAATGGTGCCTTTGGGTCT- $3^{\prime}$ ). Primer sequences for Gapdh and PCR conditions were described in previous reports. ${ }^{30}$ Expression of each mRNA was normalized by that of Gapdh mRNA.

\section{Statistical Analysis}

All data are presented as means \pm SEM. The $\chi^{2}$ test was used to measured differences in the mean intensity of positive trophoblasts on TUNEL staining between EGFP and cKO placentas. Other statistical analyses were performed using the two-tailed $t$-test. $P<0.05$ was considered significant.

\section{Results}

Systemic Atg7 Knockout Dams Deliver Pups with Fetal Growth Restriction, but Do Not Exhibit Hypertension

The effects of autophagy deficiency on the development of HDP-like symptoms in dams were first evaluated using systemic $\operatorname{Atg} 7$ knockout mice. $\operatorname{Atg} 7^{+-}$female mice were mated with $A \operatorname{tg} 7^{+/-}$male mice for the systemic knockout group, and $\operatorname{Atg} 7^{+/+}$female mice were mated with $\operatorname{Atg} 7^{+/+}$male mice for the control group. The litter size of each genotype was $1.69 \pm 0.38,4.04 \pm 0.38$, and $1.73 \pm 0.18$ for $\mathrm{Atg} 7^{+/+}$, $\operatorname{Atg} 7^{+/-}$, and $\operatorname{Atg} 7^{-1-}$, respectively, according to the mendelian ratio (Supplemental Figure S3A); and fetal body weights of $A \operatorname{tg} 7^{-1-}$ fetuses $(950 \pm 19.9 \mathrm{mg})$ were significantly lower than those of $A \operatorname{tg} 7^{+/+}$fetuses $(1135 \pm 20.4 \mathrm{mg}$; $P<0.001$ ) (Supplemental Figure S3B), as previously reported. ${ }^{19}$ Regarding dams, BP levels in $A t g 7^{+/-}$dams were comparable with those in $\mathrm{Atg} 7^{+/+}$dams at ED 18.5 (systolic $\mathrm{BP}, 107 \pm 1.2$ and $106 \pm 1.2 \mathrm{mmHg}$ in $\mathrm{Atg}^{7^{++}}$and $\mathrm{Atg} 7^{+/-}$ dams, respectively; $P=0.63$ ) (Supplemental Figure S3C). On the other hand, placental weights in $\operatorname{Atg} 7^{-1-}$ mice $(84 \pm 2.2 \mathrm{mg})$ were significantly lower than those in $\mathrm{Atg} 7^{+/+}$ mice $(92 \pm 2.6 \mathrm{mg} ; P<0.05)$ (Supplemental Figure S3D). These results suggest that systemic Atg 7 deficiency induced fetal growth restriction because of growth restriction of the placenta rather than hypertension in dams.

\section{Establishment of Placenta-Specific Atg7 Conditional Knockout Mice}

To investigate the biological significance of sole placental autophagy, placenta-specific deletion of autophagy is required to eliminate the effects of autophagy in fetuses. The unique LV vector-mediated placenta-specific gene knockout system was established because the LV vector infects only the 


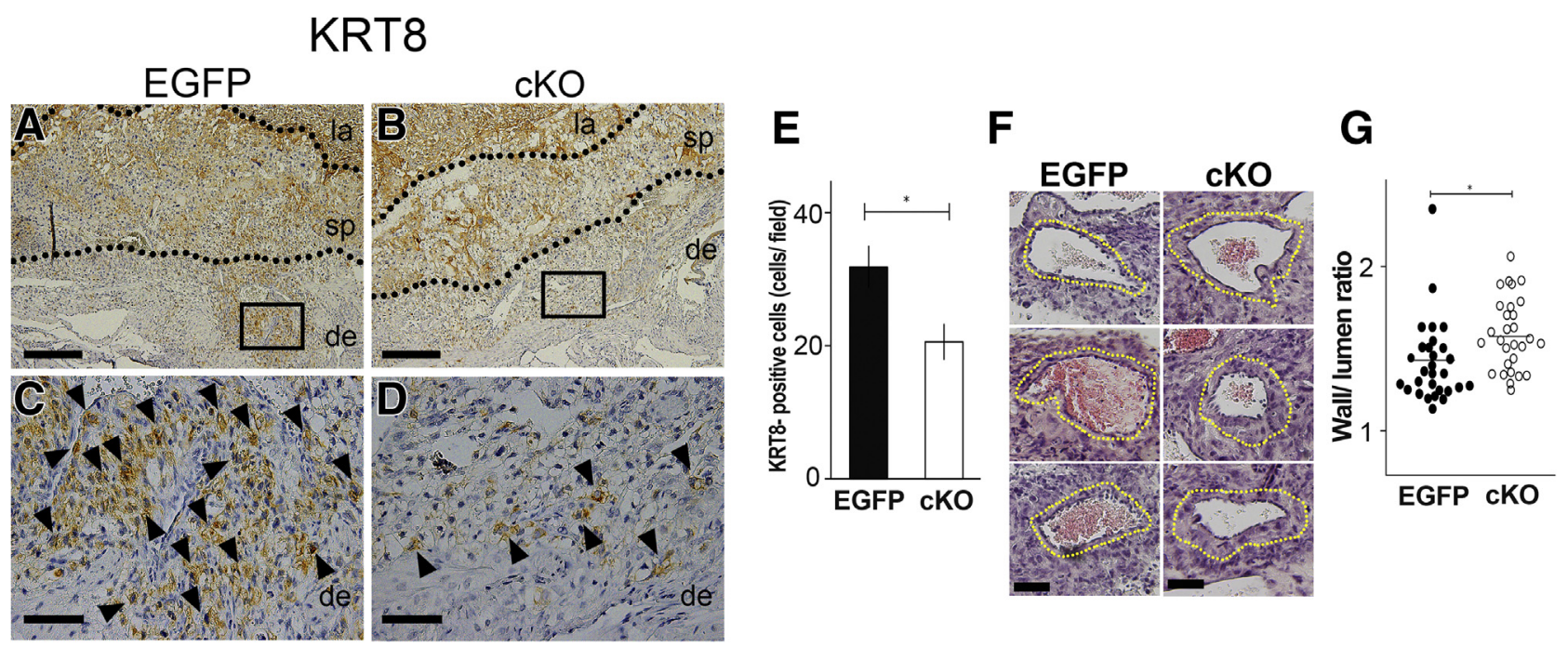

Figure 4 Failure of trophoblast invasion and vascular remodeling in placentas. A-D: Staining of keratin-8 (KRT8), which reflects the invasive phenotype of trophoblasts, was performed for EGFP and cKO placentas. A and B: The top dotted black lines indicate the borderline between labyrinth (la) and spongiotrophoblast (sp) layers, and the bottom dotted black lines indicate the borderline between spongiotrophoblast layer and decidua. C and D: The KRT8positive cells in the fetal side of the decidua (de) in the area framed by the rectangles in A and B, respectively. Arrowheads indicate KRT8-positive trophoblast cells. E: The number of KRT8-positive cells per field was counted and quantitated in the decidua in EGFP and cKO placentas. F: The representative figures show the vessels in the decidua. The vascular walls are outlined with yellow dotted lines. G: The graph shows the wall/lumen ratio in EGFP and cKO placentas to quantify the vascular remodeling of invasive trophoblasts. The lines in the middle indicate the average ratio in each group. $n=5$ EGFP (E); $n=7 \mathrm{cKO}(\mathbf{E}) ; n=29 \mathrm{EFP}$ and $\mathrm{CKO}(\mathbf{G})$. ${ }^{*} P<0.05$. Scale bars: $200 \mu \mathrm{m}(\mathbf{A}$ and $\mathbf{B}) ; 50 \mu \mathrm{m}(\mathbf{C}, \mathbf{D}$, and $\mathbf{F})$.

trophoectoderm and not the inner cell mass in blastocysts after removal of the zona pellucida. ${ }^{22}$ The Atg 7 knockout allele was only detected in placentas, confirming successful placentaspecific deletion of $\operatorname{Atg} 7$ by this system (Figure 1B). The autophagy status in placentas and fetuses was examined on the basis of SQSTM1 and MAP1LC3B proteins. Lower autophagy activity increases SQSTM1, a selective cargo protein of autophagosomes that is degraded by autophagy. When autophagy is activated, increased conversion of MAP1LC3B-I to MAP1LC3B-II is often observed with an increase in the MAP1LC3B-II/MAP1LC3B-I ratio. As a result, the depletion of Atg 7 accompanied by an increase in SQSTM1 and a decrease in the MAP1LC3B-II/MAP1LC3B-I ratio was observed in cKO placentas but not EGFP placentas by Western blot analysis (Figure 1C), suggesting that Atg7 deletion blocked the autophagy cascade in cKO placentas. These changes in autophagyrelated proteins were not noted in fetuses in either group (Figure 1C). Quantitative analyses demonstrated that the increase of SQSTM1 and the decrease in the MAP1LC3B-II/ MAP1LC3B-I ratio were significant in cKO placentas but not in fetuses (Figure 1D), confirming successful establishment of autophagy-deficient placentas by our system.

\section{HDP Phenotypes in the Placenta-Specific Atg7 Knockout Dams}

The development of HDP symptoms was seen in dams with cKO placentas. The systolic BP in wild-type dams with cKO placentas gradually elevated and remained significantly higher than that in dams with EGFP placentas from ED 16.5 (Figure 2A). Furthermore, the placental weight was significantly lower for cKO placentas than for EGFP placentas $(103 \pm 2.5$ and $92 \pm 2.3 \mathrm{mg}$ in EGFP and cKO placentas, respectively; $P<0.01$ ) (Figure 2B). Although no significant differences were observed in fetal body weights between the two groups $(1424 \pm 20.3$ or $1411 \pm 20.0 \mathrm{mg}$ in fetuses derived from EGFP or cKO placentas, respectively; $P=0.64$ ) (Figure $2 \mathrm{C}$ ), the fetal/placental weight ratio was significantly higher in the cKO group than in the EGFP group $(14.3 \pm 0.34$ and $15.5 \pm 0.36$ in EGFP and cKO, respectively; $P<0.05$ ) (Figure $2 \mathrm{D}$ ). Protein concentrations in urine in the cKO group slightly, but not significantly, increased on ED $19.5(480 \pm 53$ and $580 \pm 60 \mathrm{ng} / \mathrm{mL}$ in dams with EGFP and cKO placentas, respectively; $P=0.20$ ) (Figure 2E). These results indicate that placentaspecific autophagy deficiency naturally induced hypertension but not proteinuria or fetal growth restriction in dams.

\section{Reduction in the Spongiotrophoblast Layer Volume with Accumulation of SQSTM1 in cKO Placentas}

The layers susceptible to autophagy inhibition leading to placental growth restriction were next investigated in $\mathrm{cKO}$ placentas. The proportion of the spongiotrophoblast layer in cKO placentas/the whole placenta area was significantly lower than that in EGFP placentas $(28.5 \% \pm 2.7 \%$ and $22.0 \% \pm 1.2 \%$ in EGFP and cKO, respectively; $P<0.05$ ) (Figure 3, A and B). On the other hand, the proportion of the labyrinth layer/the whole placental area was comparable between the two groups $(64.2 \% \pm 3.1 \%$ and $68.3 \% \pm 1.9 \%$ in EGFP and cKO, respectively; $P=0.25$ ) (Figure 3, A and B). Consistent with the results of Western blot analysis 


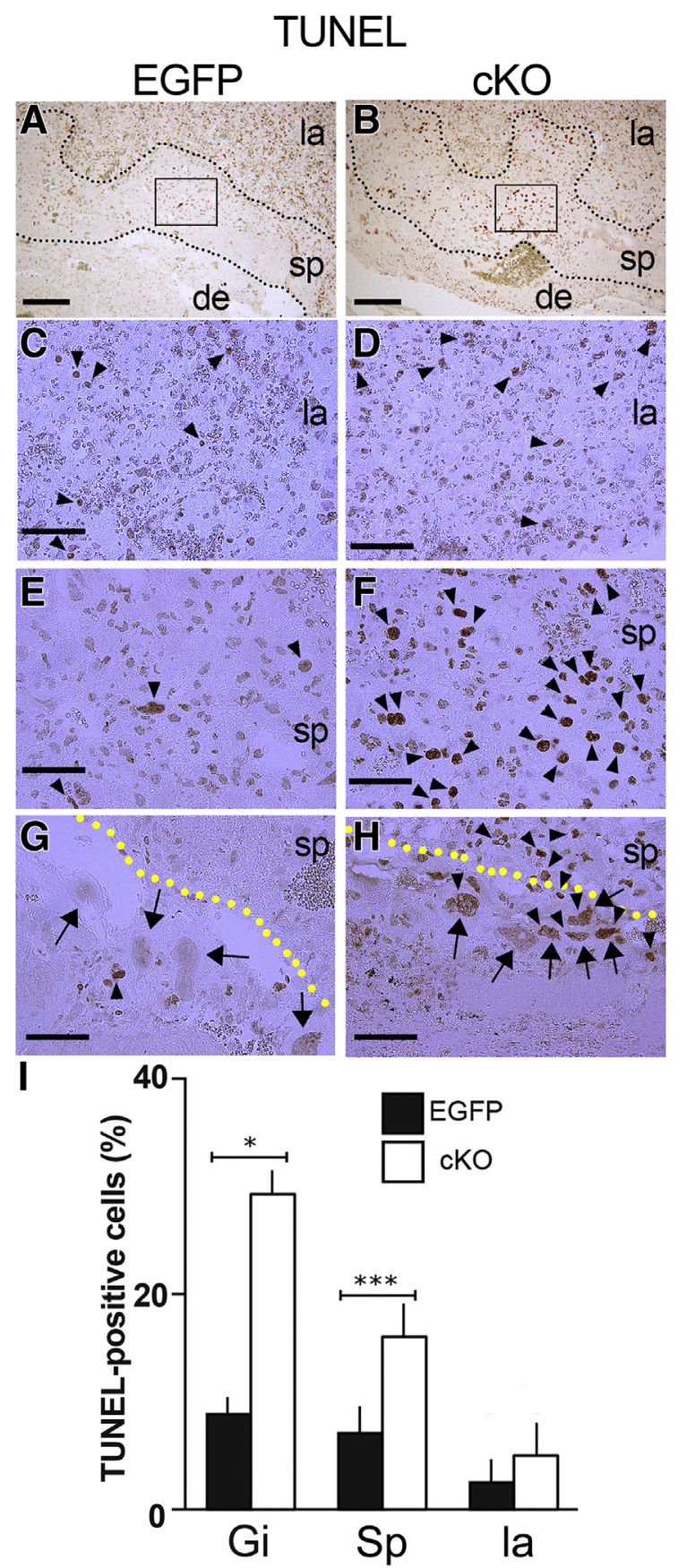

Figure 5 Apoptosis in the spongiotrophoblast layer in autophagydeficient placentas. A and B: Trophoblast apoptosis in the EGFP or cKO placentas, as analyzed by terminal deoxynucleotidyl transferase-mediated dUTP nick end labeling (TUNEL) assay. A and B: The top dotted black lines indicate the borderline between the labyrinth and spongiotrophoblast layers, and the bottom dotted black lines indicate the borderline between the spongiotrophoblast layer and decidua (de). C-H: TUNEL-positive cells were observed in the labyrinth (la) layer ( $\mathbf{C}$ and $\mathbf{D}$ ), the spongiotrophoblast (sp) layer ( $\mathbf{E}$ and $\mathbf{F})$, and the giant trophoblast layer ( $\mathbf{G}$ and $\mathbf{H})$. $\mathbf{E}$ and $\mathbf{F}$ : The boxed areas from $\mathbf{A}$ and $\mathbf{B}$, shown at higher magnification. $\mathbf{G}$ and $\mathbf{H}$ : The dotted yellow lines indicate the borderline between spongiotrophoblast layer and decidua. Arrowheads indicate TUNEL-positive cells; arrows, parietal trophoblast giant cells. I: The graph shows the proportion of TUNELpositive cells in the labyrinth layer, spongiotrophoblast layer, and giant trophoblast cells (Gi) in EGFP and cK0 placentas. ${ }^{*} P<0.05,{ }^{* * *} P<0.001$. Scale bars: $200 \mu \mathrm{m}(\mathbf{A}$ and $\mathbf{B}) ; 50 \mu \mathrm{m}(\mathbf{C}-\mathbf{H})$.
(Figure 1C), a significant reduction in Atg7 expression was observed in cKO placentas (Figure 3, F-H) compared with in EGFP placentas (Figure 3, C-E). However, the Atg7 expression level among the labyrinth layer, spongiotrophoblast layer, and parietal trophoblast giant cells did not differ in the EGFP placentas (Figure 3, C-E). The placental layer in which autophagy activity was highly suppressed was next evaluated on the basis of the accumulation of SQSTM1, which is mainly degraded by autophagy machinery, by immunohistochemistry. Staining of SQSTM1 was higher in the spongiotrophoblast layer in cKO placentas (Figure 3M) than that in EGFP placentas (Figure $3 \mathbf{J}$ ). In addition, similar results were observed in the parietal trophoblast giant cells in cKO (Figure 3N) and EGFP (Figure 3K) placentas. On the other hand, SQSTM1 was expressed in the labyrinth layer in both groups (Figure 3, I and L). Taken together, spongiotrophoblasts and parietal trophoblast giant cells may be susceptible to autophagy deficiency, resulting in the suppression of spongiotrophoblast layer development.

\section{Impairment of Trophoblast Cell Invasion and Vascular Remodeling in cKO Placentas}

To clarify the cause of the smaller size of cKO placentas, which is similar with poor placentation in human preeclamptic placentas, the invasive ability of trophoblasts into the decidua was evaluated by counting the number of keratin-8-positive cells on the fetal side of the decidua in EGFP and cKO placentas (Figure 4, A-D). Impairment of invasion and vascular remodeling in trophoblasts leads to poor placentation in the first step of PE. Quantitative analysis demonstrated that the number of trophoblasts that had detached from the spongiotrophoblast layer was significantly reduced in cKO placentas at ED 19.5 $(31.8 \pm 3.1$ and $20.5 \pm 2.7$ per field in EGFP and cKO placentas, respectively; $P<0.05$ ) (Figure 4E). Vascular remodeling was also evaluated by the wall/lumen ratio of spiral arteries, as previously described in other murine models of $\mathrm{PE}^{31,32}$ The ratio in cKO placentas was significantly increased compared with that in EGFP placentas at ED $19.5(1.36 \pm 0.045$ and $1.53 \pm 0.040$ in EGFP and cKO placentas, respectively; $P<0.05$ ) (Figure 4, F and G), indicating impaired vascular remodeling of the spiral arteries in cKO placentas. Furthermore, the invasion assay using a rodent trophoblast cell line, Rcho-1, demonstrated that the invasive ability was significantly reduced when autophagy was suppressed by BAF in a dose-dependent manner (Supplemental Figure S2A). These results were similar with the reported invasive behavior of human trophoblast cell lines. ${ }^{9,27}$ However, the invasive ability was increased when the autophagy activity was activated by TRN1, a specific inhibitor of mammalian target of rapamycin. Consistent with the results of invasion, pro-matrix metalloproteinase 2 and 9 activity was significantly reduced in the presence of $20 \mathrm{nmol} / \mathrm{L}$ $\mathrm{BAF}$, but increased in the presence of $20 \mathrm{nmol} / \mathrm{L}$ TRN1 (Supplemental Figure S2, B and C). Therefore, autophagy activity may regulate invasion in rodent trophoblast cells. 

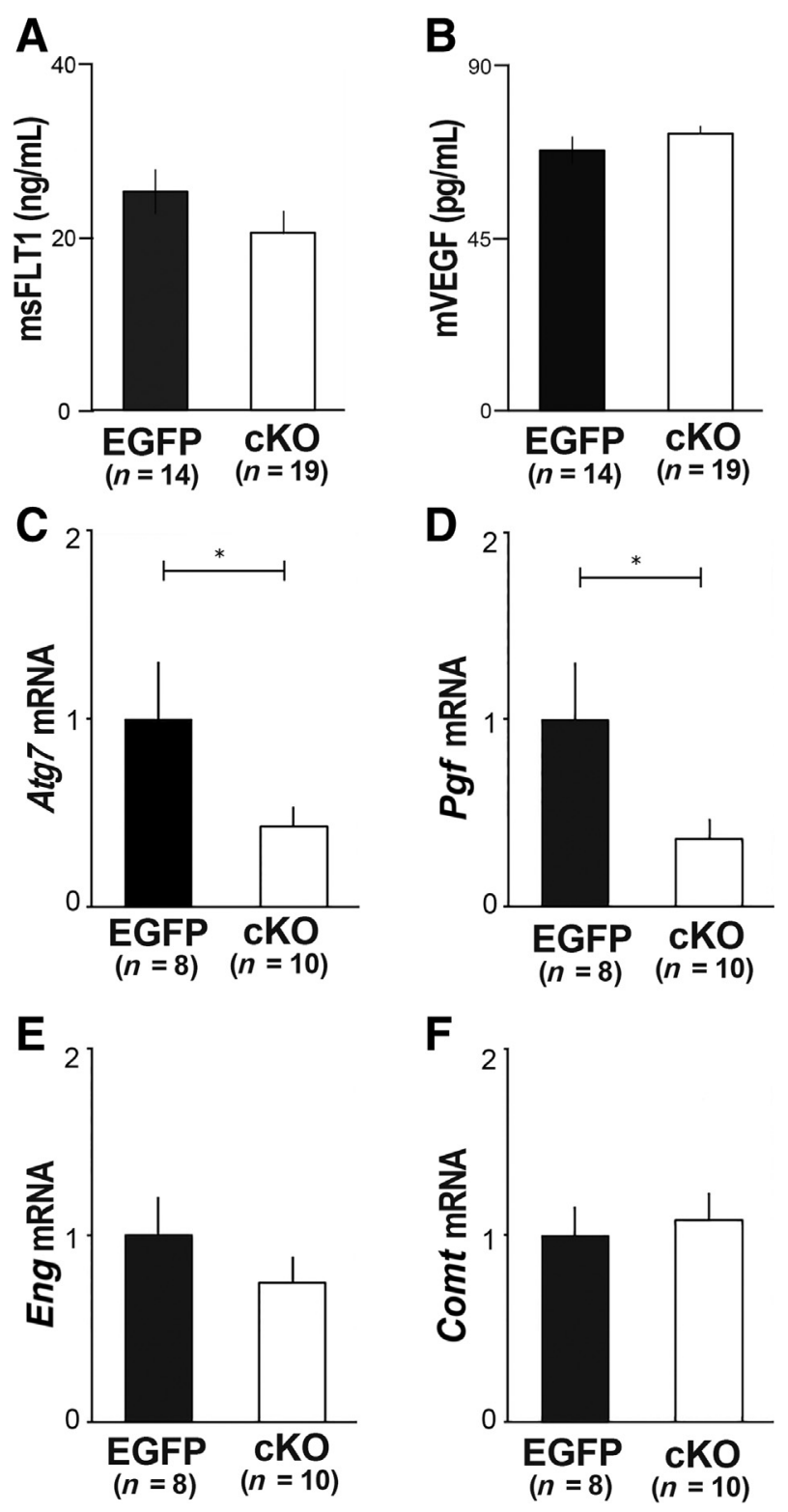

Figure 6 mRNA expression of hypertensive disorder of pregnancy-related factors in autophagy-deficient placentas. A: The bars indicate the mouse sFLT1 (msFLT1) concentration in serum obtained from dams bearing EGFP or CKO placentas at embryonic day (ED) 18.5. B: The bars indicate the mouse vascular endothelial growth factor (mVEGF) concentration in serum obtained from dams bearing EGFP or CKO placentas at ED 18.5. C-F: The bars indicate the relative expression levels of $\operatorname{Atg} 7$ (C), Pgf (D), Eng (E), and Comt (F) mRNA. Data were normalized to Gapdh mRNA levels in each placenta type. ${ }^{*} P<0.05$.

\section{An Increase in Cell Death in the Spongiotrophoblast Layer and Trophoblast Giant Cells in cKO Placentas}

To clarify the other effects of autophagy deficiency in placentas, TUNEL staining was performed to detect apoptotic cells in each placental layer (Figure 5, A and B). TUNELpositive cells composed $7.0 \%$ in EGFP placentas, whereas those in $\mathrm{cKO}$ placentas composed $16.0 \%$ of the spongiotrophoblast layer $(P<0.001)$ (Figure 5, E, F, and I). Among the parietal trophoblast giant cells, the percentage of TUNELpositive cells was also significantly increased to $29.2 \%$ in cKO placentas compared with $8.8 \%$ in EGFP placentas $(P<0.05)$ (Figure 5, G-I). However, in the labyrinth layer, the percentages in EGFP and cKO placentas were similar (2.5\% and 5.0\%, respectively) (Figure 5, C, D, and I). To determine other causes of cell death in the trophoblasts, several factors related to HDP, such as hypoxia-inducible factor- $1 \alpha$ and the oxidative stress marker 8 -hydroxy-2'deoxyguanosine, were examined (Supplemental Figure S4), but there were no significant differences between EGFP and cKO placentas. Taken together, autophagy deficiency may mediate cell death, thereby reducing the spongiotrophoblast layer or parietal trophoblast giant cell area in cKO placentas.

\section{Decrease of Pgf mRNA in the cKO Placentas}

To clarify other causes of poor placentation, the serum level of mouse sFLT1 was measured in dams because an increase of sFLT1 in human serum, which disrupts endothelial function, is involved in hypertension and proteinuria in preeclamptic women. The sFLT1 level in dams with cKO placentas was similar to that in dams with EGFP placentas $(25.5 \pm 2.5$ and $20.4 \pm 2.5 \mathrm{ng} / \mathrm{mL}$ in dams with EGFP and cKO placentas, respectively; $P=0.22$ ) (Figure 6A). The serum level of mouse VEGF was also similar between the two groups $(67.7 \pm 3.5$ and $72.1 \pm 1.9 \mathrm{pg} / \mathrm{mL}$ in dams with EGFP and cKO placentas, respectively; $P=0.35$ ) (Figure 6B). The relative mRNA expression of the PE-related factors Eng, $C o m t$, and $P g f$ was measured in placentas. COMT-deficient pregnant mice have been reported to exhibit hypertension and proteinuria by induced placental hypoxia and SFLT1 elevation. ${ }^{31}$ First, reduced Atg7 mRNA was confirmed in the $\mathrm{cKO}$ placentas as an internal control $(P<0.01)$ (Figure 6C). Next, the relative $P g f$ mRNA expression was significantly suppressed in cKO placentas $(1.0 \pm 0.30$ versus $0.36 \pm 0.10$; $P<0.05$ ) (Figure 6D). Relative mRNA expression of Eng and Comt, however, was not significantly different between EGFP and cKO placentas (Figure 6, E and F). These results suggest that the growth restriction in autophagy-deficient placentas was mediated by PGF reduction.

\section{Discussion}

The LV-mediated placenta-specific Atg7 knockout system enabled us to investigate the effects of placental autophagy deficiency separately from fetal autophagy deficiency because Atg7, an E1-like activating enzyme that facilitates both the conjugation of phosphatidylethanolamine to MAP1LC3B and ATG12-ATG5 conjugation, acts as the hub of these two essential systems for autophagosome formation. ${ }^{19}$ Before this experiment, lentivirus-mediated overexpression of $\mathrm{Atg} 4 \mathrm{~B}^{\mathrm{C} 74 \mathrm{~A}}$ mutant protein, which strongly inhibits autophagy by blocking phosphatidylethanolamine conjugation to MAP1LC3B ${ }^{33}$ did not inhibit autophagy in 


\section{Pathophysiology of preeclampsia and autophagy} [two-stage disorder]

First stage

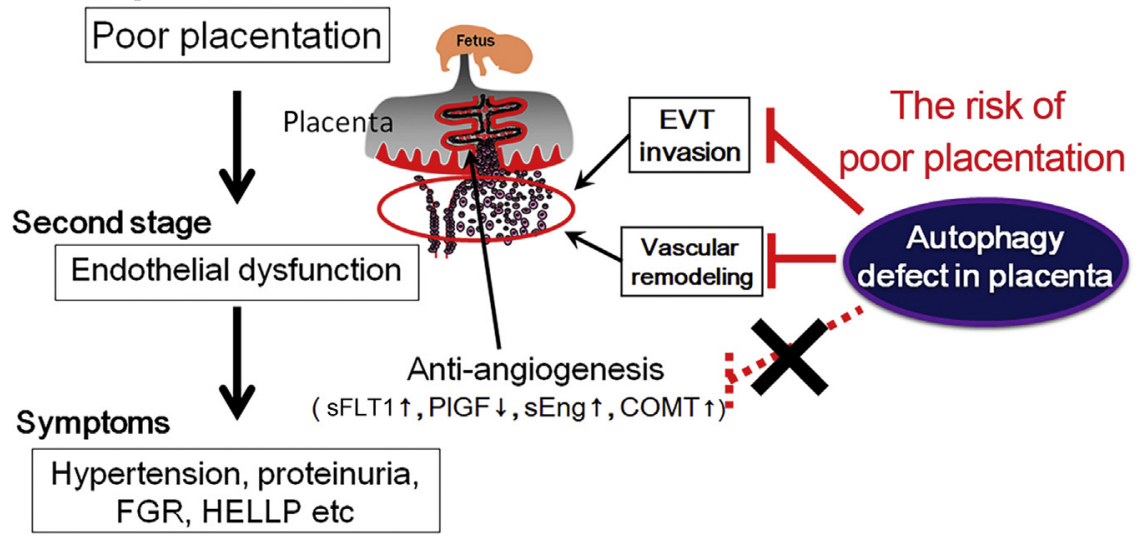

Figure 7 Inhibition of autophagy in placentas is a risk factor for poor placentation. Two fundamental functions of extravillous trophoblasts (EVTs), adequate invasion and vascular remodeling, are required for normal placentation in early pregnancy. Autophagy deficiency in placentas inhibits EVT function, resulting in poor placentation at the first stage. On the other hand, the production of antiangiogenic factors from placentas, which is mediated by endothelial dysfunction at the second stage, does not directly correlate with autophagy deficiency in placentas. The inhibition of autophagy in placentas is one risk factor for poor placentation. COMT, catechol-0methyltransferase; FGR, fetal growth restriction; HELLP, hemolysis, elevated liver enzyme, and low platelet syndrome; PIGF, placental growth facotor; sEng, soluble endoglin. placentas (data not shown). Subsequently, this study demonstrated that placental growth is restricted by Atg7 deficiency in placentas, which also induced hypertension in dams. However, fetal growth restriction was not observed in pups with normal autophagy. Regarding placental development, shallow trophoblast invasion and poor vascular remodeling of spiral arteries, features of the first step of PE pathophysiology observed in cKO placentas, led to placental growth restriction (Figure 7). The accumulation of SQSTM1 was seen in the invading trophoblasts around spiral arteries as well as central canals (Supplemental Figure S5). In addition, increased apoptosis in the spongiotrophoblast layer and parietal trophoblast giant cells was consistent with the accumulation of SQSTM1, a marker of autophagy inhibition, in cKO placentas, suggesting that autophagy inhibition also mediates cell death in murine trophoblasts. Regarding human invasive trophoblasts, SQSTM1 accumulates in the EVTs in placental tissue samples of PE, as well as in two autophagydeficient EVT cell lines in which autophagy is constitutively inhibited by ATG4B ${ }^{\mathrm{C} 74 \mathrm{~A}}$ mutation. ${ }^{9,33}$ Thus, cKO placentas exhibited similar phenotypes of autophagy inhibition as human preeclampsia placentas, suggesting SQSTM1 to be an indicator of autophagy inhibition in the placenta.

On the basis of the two-stage model of $\mathrm{PE},{ }^{1,2}$ poor placentation is attributable to shallow trophoblast invasion and failure of vascular remodeling as the first step. There are several reports stating that excessive autophagy activation is involved in the pathophysiology of PE. ${ }^{13,14,34,35}$ In contrast, we previously proposed that impaired autophagy in human trophoblasts inhibited EVT invasion and vascular remodeling, resulting in PE. ${ }^{9,27,36,37}$ Thus, a discrepancy exists. Mizushima et $\mathrm{al}^{11}$ reported unsuccessful measurement of autophagic flux in fixed tissues and that the common markers for autophagy do not work in tissue sections. Therefore, these placenta-specific autophagy-deficient mice are necessary to precisely evaluate the role of autophagy for placentation. Regarding diseases related to autophagy, many studies have found that autophagy inhibition induces dysfunction in several organs, such as the central nervous system and liver. ${ }^{38-41}$ Compared with studies using systemic Atg7 knockout mice, this study using cKO placentas more strongly supports the importance of autophagy inhibition for both trophoblastic dysfunction and high blood pressure in dams (Supplemental Table S2). In addition, in vitro analysis using Rcho-1, a murine trophoblast cell line, revealed that their invasive ability was significantly reduced when autophagy was suppressed by BAF, but was restored by TRN1 (Supplemental Figure S2A). Thus, autophagy inhibition may directly induce poor placentation via dysfunction of invasive trophoblasts in mice and humans. In addition to the SQSTM1 protein, other autophagy-related proteins that reflect autophagy inhibition in placental samples are needed to more precisely estimate autophagy activity in placentas because it varies among organs.

As the second step of the two-stage model of PE, sFLT1, soluble endoglin, PGF, VEGF, and COMT have been found to correlate with the pathophysiology of PE because of endothelial dysfunction. ${ }^{3-5,32}$ The correlations between these factors and autophagy were thus examined. A significant reduction in $P g f$ mRNA levels was only noted in cKO placentas, but sFLT1 and VEGF concentrations in the maternal serum and Eng and Comt mRNA levels in cKO placentas were unchanged (Figure 6). Although the endothelial dysfunction induced by antiangiogenic agents often clinically appears as proteinuria and hypertension in dams, hypertension was only observed in dams with cKO placentas. Thus, autophagy deficiency in trophoblasts is likely not correlated with the production of antiangiogenic factors in the second step (Figure 7), but it is still unknown how autophagy deficiency decreases $P g f$ mRNA expression. Further studies are required to clarify this question. On the other hand, autophagy inhibition impairs VEGF-mediated angiogenesis without altered VEGF expression in aortic endothelial cells. ${ }^{42}$ Moreover, it was reported that the antiangiogenic effects of bevacizumab, a monoclonal antibody against VEGF-like 
sFLT1, were enhanced in tumor cells with autophagy inhibition. ${ }^{43,44}$ Therefore, placental autophagy deficiency not only elicits poor placentation, but may also sensitize placentas to antiangiogenic agents because of the disruption of homeostasis in trophoblasts. In addition, regarding measurement of BP, a sphygmomanometer was used instead of a telemetry system in this study. To obtain more accurate BP data (ie, comparing BP between day and night), a telemetry system is more beneficial to examine physiological changes.

LV-EGFP was found to be uniformly expressed in the labyrinth trophoblast layer, the spongiotrophoblast layer, and the parietal trophoblast giant cells in the original report of the LV gene expression system. ${ }^{22}$ However, in our experiment, SQSTM1 was mostly accumulated in the spongiotrophoblast layer and parietal trophoblast giant cells in cKO placentas, but the expression level of SQSTM1 in the labyrinth layer in cKO and EFGP placentas was similar (Figure 3, I and L). One reason as to why SQSTM1 accumulated in the parietal trophoblast giant cells and spongiotrophoblast layer in $\mathrm{cKO}$ placentas is that the labyrinth layer may be resistant to autophagy inhibition because syncytiotrophoblasts (STBs), which are comparable to murine trophoblasts in the labyrinth layer, have higher autophagy activity than cytotrophoblasts in humans. ${ }^{45}$ STBs are more resistant than cytotrophoblasts to bacteria, which are surrounded and eliminated in cells by cellular autophagy. ${ }^{46}$ Although there are structural differences between human and mouse placentas, mouse giant trophoblasts, which localize in the outer layer in the placenta and face maternal cells like human EVTs, may require energy from autophagy because human EVTs require adenosine triphosphates produced by autophagy during invasion. ${ }^{27}$ Indeed, greater SQSTM1 accumulation, mainly in EVTs, in human PE placentas than in normal placentas has been previously reported. $^{9}$

In contrast with the placenta-specific Atg7 knockout, Muralimanoharan et $\mathrm{al}^{47}$ established STB-specific Atg7 knockout placentas by crossbreeding $A \operatorname{tg} 7^{\text {flox/flox }}$ mice with Cre-recombinase transgenic mice driven by cytochrome $\mathrm{P} 450$ family 19 subfamily A member 1 (CYP19A1), which is restricted to STBs in the labyrinth layer, resulting in impaired autophagic activity. The placental weight did not differ between control and conditional knockout placentas, but fetal growth restriction was markedly observed in fetuses from the STB-specific conditional knockout mice. Our placentaspecific Atg7 knockout inhibited placental but not fetal growth; however, STB-specific Atg7 knockout inhibited fetal but not placental growth. Therefore, it is difficult to directly compare our results because the deletion of autophagy occurred in different placental layers. Autophagy suppression in spongiotrophoblasts or parietal trophoblast giant cells may affect placental growth rather than fetal growth because of impairment of invasion or vascular remodeling of invasive trophoblasts. On the other hand, autophagy suppression in STBs in the labyrinth layer, which is responsible for fetal nutrition, may affect fetal growth rather than placental growth. In addition, the autophagy suppression in spongiotrophoblasts and parietal trophoblast giant cells in this study induced gestational hypertension because of placental growth restriction in dams. The fetal/placental weight ratio, which is often used as an index of placental nutrient efficiency, was significantly higher in the placenta-specific Atg7 knockout placentas than in EGFP placentas, but it was lower in the STB-specific Atg7 knockout placentas. In general, a high fetal/placental weight ratio increases the risk of poor pregnancy outcome or development of hypertension later in life. $^{48,49}$ Thus, the high fetal/placental weight ratio, which was attributable to placental growth restriction, correlated with the risk of developing hypertension in dams.

This is the first report demonstrating that autophagy deficiency in trophoblasts correlates with poor placentation because of inadequate vascular remodeling and invasion, and induces hypertension in dams. On the other hand, although it has no direct effects on endothelial dysfunction, it may sensitize placentas to antiangiogenic agents because of the disruption of homeostasis in trophoblasts. HDP is not a singleetiological disease and, thus, autophagy explains possibly a part of HDP pathogenesis/etiology in that placental autophagy deficiency may lead to HDP because of poor placentation.

\section{Acknowledgments}

We thank Prof. Masaaki Komatsu (Niigata University, Japan) for gifting the Atg7 knockout mice, Prof. Hiroki Shoji (Kanazawa Medical University) and Prof. Toshihisa Hatta (Kanazawa Medical University) for advising on Rcho1 cell culture, and Prof. Michael J. Soares (University of Kansas Medical Center) for providing Rcho-1 cells.

A.A. performed most experiments and wrote the article; A.N. performed the autophagy experiments with the Rcho-1 trophoblast cell line and edited the article; T.K. provided immunohistochemistry results; Y.O. and O.Y. provided quantitative RT-PCR data; M.M., K.K., and M.I. helped to establish the placenta-specific Atg7-knockout mice; T.Y. provided advice on autophagy experiments and edited the article; S.S. served as the principal investigator of this study, supervised the project for its overall design and concept, analyzed the data, and wrote the article.

\section{Supplemental Data}

Supplemental material for this article can be found at https://doi.org/10.1016/j.ajpath.2018.07.021.

\section{References}

1. Redman CW, Sargent IL: Immunology of pre-eclampsia. Am J Reprod Immunol 2010, 63:534-543

2. Steegers EA, von Dadelszen P, Duvekot JJ, Pijnenborg R: Preeclampsia. Lancet 2010, 376:631-644

3. Levine RJ, Lam C, Qian C, Yu KF, Maynard SE, Sachs BP, Sibai BM, Epstein FH, Romero R, Thadhani R, Karumanchi SA: Soluble 
endoglin and other circulating antiangiogenic factors in preeclampsia. N Engl J Med 2006, 355:992-1005

4. Levine RJ, Maynard SE, Qian C, Lim KH, England LJ, Yu KF, Schisterman EF, Thadhani R, Sachs BP, Epstein FH, Sibai BM, Sukhatme VP, Karumanchi SA: Circulating angiogenic factors and the risk of preeclampsia. N Engl J Med 2004, 350:672-683

5. Venkatesha S, Toporsian M, Lam C, Hanai J, Mammoto T, Kim YM, Bdolah Y, Lim KH, Yuan HT, Libermann TA, Stillman IE, Roberts D, D'Amore PA, Epstein FH, Sellke FW, Romero R, Sukhatme VP, Letarte M, Karumanchi SA: Soluble endoglin contributes to the pathogenesis of preeclampsia. Nat Med 2006, 12:642-649

6. Benyo DF, Smarason A, Redman CW, Sims C, Conrad KP: Expression of inflammatory cytokines in placentas from women with preeclampsia. J Clin Endocrinol Metab 2001, 86:2505-2512

7. Maynard SE, Min JY, Merchan J, Lim KH, Li J, Mondal S, Libermann TA, Morgan JP, Sellke FW, Stillman IE, Epstein FH, Sukhatme VP, Karumanchi SA: Excess placental soluble fms-like tyrosine kinase 1 (sFlt1) may contribute to endothelial dysfunction, hypertension, and proteinuria in preeclampsia. J Clin Invest 2003, 111: 649-658

8. Walker JJ: Antioxidants and inflammatory cell response in preeclampsia. Semin Reprod Endocrinol 1998, 16:47-55

9. Nakashima A, Yamanaka-Tatematsu M, Fujita N, Koizumi K, Shima T, Yoshida T, Nikaido T, Okamoto A, Yoshimori T, Saito S: Impaired autophagy by soluble endoglin, under physiological hypoxia in early pregnant period, is involved in poor placentation in preeclampsia. Autophagy 2013, 9:303-316

10. Mizushima N, Komatsu M: Autophagy: renovation of cells and tissues. Cell 2011, 147:728-741

11. Mizushima N, Yoshimori T, Levine B: Methods in mammalian autophagy research. Cell 2010, 140:313-326

12. Hung TH, Hsieh TT, Chen SF, Li MJ, Yeh YL: Autophagy in the human placenta throughout gestation. PLoS One 2013, 8:e83475

13. Kalkat M, Garcia J, Ebrahimi J, Melland-Smith M, Todros T, Post M, Caniggia I: Placental autophagy regulation by the BOK-MCL1 rheostat. Autophagy 2013, 9:2140-2153

14. Melland-Smith M, Ermini L, Chauvin S, Craig-Barnes H, Tagliaferro A, Todros T, Post M, Caniggia I: Disruption of sphingolipid metabolism augments ceramide-induced autophagy in preeclampsia. Autophagy 2015, 11:653-669

15. Nakashima A, Aoki A, Kusabiraki T, Cheng SB, Sharma S, Saito S: Autophagy regulation in preeclampsia: pros and cons. J Reprod Immunol 2017, 123:17-23

16. Nakashima A, Aoki A, Kusabiraki T, Shima T, Yoshino O, Cheng SB, Sharma S, Saito S: Role of autophagy in oocytogenesis, embryogenesis, implantation, and pathophysiology of pre-eclampsia. J Obstet Gynaecol Res 2017, 43:633-643

17. Kim J, Dalton VM, Eggerton KP, Scott SV, Klionsky DJ: Apg $7 \mathrm{p} / \mathrm{Cvt} 2 \mathrm{p}$ is required for the cytoplasm-to-vacuole targeting, macroautophagy, and peroxisome degradation pathways. Mol Biol Cell 1999, 10:1337-1351

18. Tanida I, Mizushima N, Kiyooka M, Ohsumi M, Ueno T, Ohsumi Y, Kominami E: Apg7p/Cvt2p: a novel protein-activating enzyme essential for autophagy. Mol Biol Cell 1999, 10:1367-1379

19. Komatsu M, Waguri S, Ueno T, Iwata J, Murata S, Tanida I, Ezaki J, Mizushima N, Ohsumi Y, Uchiyama Y, Kominami E, Tanaka K, Chiba T: Impairment of starvation-induced and constitutive autophagy in Atg7-deficient mice. J Cell Biol 2005, 169:425-434

20. Kuma A, Hatano M, Matsui M, Yamamoto A, Nakaya H, Yoshimori T, Ohsumi Y, Tokuhisa T, Mizushima N: The role of autophagy during the early neonatal starvation period. Nature 2004, 432:1032-1036

21. Morioka Y, Isotani A, Oshima RG, Okabe M, Ikawa M: Placenta-specific gene activation and inactivation using integrase-defective lentiviral vectors with the Cre/LoxP system. Genesis 2009, 47:793-798

22. Okada Y, Ueshin Y, Isotani A, Saito-Fujita T, Nakashima H, Kimura K, Mizoguchi A, Oh-Hora M, Mori Y, Ogata M, Oshima RG,
Okabe M, Ikawa M: Complementation of placental defects and embryonic lethality by trophoblast-specific lentiviral gene transfer. Nat Biotechnol 2007, 25:233-237

23. Kumasawa K, Ikawa M, Kidoya H, Hasuwa H, Saito-Fujita T, Morioka Y, Takakura N, Kimura T, Okabe M: Pravastatin induces placental growth factor (PGF) and ameliorates preeclampsia in a mouse model. Proc Natl Acad Sci U S A 2011, 108:1451-1455

24. Nakashima A, Shiozaki A, Myojo S, Ito M, Tatematsu M, Sakai M, Takamori Y, Ogawa K, Nagata K, Saito S: Granulysin produced by uterine natural killer cells induces apoptosis of extravillous trophoblasts in spontaneous abortion. Am J Pathol 2008, 173:653-664

25. Arikawa T, Liao S, Shimada H, Inoue T, Sakata-Haga H, Nakamura T, Hatta T, Shoji H: Galectin-4 expression is down-regulated in response to autophagy during differentiation of rat trophoblast cells. Sci Rep 2016, 6:32248

26. Faria TN, Soares MJ: Trophoblast cell differentiation: establishment, characterization, and modulation of a rat trophoblast cell line expressing members of the placental prolactin family. Endocrinology 1991, 129:2895-2906

27. Yamanaka-Tatematsu M, Nakashima A, Fujita N, Shima T, Yoshimori T, Saito S: Autophagy induced by HIF1alpha overexpression supports trophoblast invasion by supplying cellular energy. PLoS One 2013, 8:e76605

28. Yoshino O, Izumi G, Shi J, Osuga Y, Hirota Y, Hirata T, Harada M, Nishii O, Koga K, Taketani Y: Activin-A is induced by interleukin-1beta and tumor necrosis factor-alpha and enhances the mRNA expression of interleukin- 6 and protease-activated receptor- 2 and proliferation of stromal cells from endometrioma. Fertil Steril 2011, 96:118-121

29. Yoshino O, Osuga Y, Hirota Y, Koga K, Hirata T, Harada M, Morimoto C, Yano T, Nishii O, Tsutsumi O, Taketani Y: Possible pathophysiological roles of mitogen-activated protein kinases (MAPKs) in endometriosis. Am J Reprod Immunol 2004, 52:306-311

30. Yosuke O, Miwako N, Osamu Y, Kaori K, Allah N, Hideki H, Hirofumi N, Gentaro I, Akitoshi N, Johji I, Kazuyuki T, Tomoyuki F, Yutaka O, Shigeru S: CD11c+ M1-like macrophages (MФs) but not CD206+ M2-like M $\Phi$ are involved in folliculogenesis in mice ovary. Sci Rep 2018, 8:8171

31. Croy BA, Burke SD, Barrette VF, Zhang J, Hatta K, Smith GN, Bianco J, Yamada AT, Adams MA: Identification of the primary outcomes that result from deficient spiral arterial modification in pregnant mice. Pregnancy Hypertens 2011, 1:87-94

32. Kanasaki K, Palmsten K, Sugimoto H, Ahmad S, Hamano Y, Xie L, Parry S, Augustin HG, Gattone VH, Folkman J, Strauss JF, Kalluri R: Deficiency in catechol-O-methyltransferase and 2-methoxyoestradiol is associated with pre-eclampsia. Nature 2008, 453:1117-1121

33. Fujita N, Hayashi-Nishino M, Fukumoto H, Omori H, Yamamoto A, Noda T, Yoshimori T: An Atg4B mutant hampers the lipidation of LC3 paralogues and causes defects in autophagosome closure. Mol Biol Cell 2008, 19:4651-4659

34. Gao L, Qi HB, Kamana KC, Zhang XM, Zhang H, Baker PN: Excessive autophagy induces the failure of trophoblast invasion and vasculature: possible relevance to the pathogenesis of preeclampsia. J Hypertens 2015, 33:106-117

35. Pan T, He G, Chen M, Bao C, Chen Y, Liu G, Zhou M, Li S, Xu W, Liu X: Abnormal CYP11A1 gene expression induces excessive autophagy, contributing to the pathogenesis of preeclampsia. Oncotarget 2017, 8:89824-89836

36. Saito S, Nakashima A: Review: the role of autophagy in extravillous trophoblast function under hypoxia. Placenta 2013, 34 Suppl: S79-S84

37. Saito S, Nakashima A: A review of the mechanism for poor placentation in early-onset preeclampsia: the role of autophagy in trophoblast invasion and vascular remodeling. J Reprod Immunol 2014, 101-102:80-88

38. Hara T, Nakamura K, Matsui M, Yamamoto A, Nakahara Y, SuzukiMigishima R, Yokoyama M, Mishima K, Saito I, Okano H, Mizushima N: Suppression of basal autophagy in neural cells causes neurodegenerative disease in mice. Nature 2006, 441:885-889 
39. Komatsu M, Waguri S, Chiba T, Murata S, Iwata J, Tanida I, Ueno T, Koike M, Uchiyama Y, Kominami E, Tanaka K: Loss of autophagy in the central nervous system causes neurodegeneration in mice. Nature 2006, 441:880-884

40. Levine B, Kroemer G: Autophagy in the pathogenesis of disease. Cell 2008, 132:27-42

41. Tanaka S, Hikita H, Tatsumi T, Sakamori R, Nozaki Y, Sakane S, Shiode Y, Nakabori T, Saito Y, Hiramatsu N, Tabata K, Kawabata T, Hamasaki M, Eguchi $H$, Nagano $H$, Yoshimori $T$, Takehara $T$ : Rubicon inhibits autophagy and accelerates hepatocyte apoptosis and lipid accumulation in nonalcoholic fatty liver disease in mice. Hepatology 2016, 64:1994-2014

42. Du J, Teng RJ, Guan T, Eis A, Kaul S, Konduri GG, Shi Y: Role of autophagy in angiogenesis in aortic endothelial cells. Am J Physiol Cell Physiol 2012, 302:C383-C391

43. Guo XL, Li D, Sun K, Wang J, Liu Y, Song JR, Zhao QD, Zhang SS, Deng WJ, Zhao X, Wu MC, Wei LX: Inhibition of autophagy enhances anticancer effects of bevacizumab in hepatocarcinoma. J Mol Med (Berl) 2013, 91:473-483
44. Selvakumaran M, Amaravadi RK, Vasilevskaya IA, O'Dwyer PJ: Autophagy inhibition sensitizes colon cancer cells to antiangiogenic and cytotoxic therapy. Clin Cancer Res 2013, 19:2995-3007

45. Cao B, Macones C, Mysorekar IU: ATG16L1 governs placental infection risk and preterm birth in mice and women. JCI Insight 2016, 1:e86654

46. Nakagawa I, Amano A, Mizushima N, Yamamoto A, Yamaguchi H, Kamimoto T, Nara A, Funao J, Nakata M, Tsuda K, Hamada S, Yoshimori T: Autophagy defends cells against invading group A Streptococcus. Science 2004, 306:1037-1040

47. Muralimanoharan S, Gao X, Weintraub S, Myatt L, Maloyan A: Sexual dimorphism in activation of placental autophagy in obese women with evidence for fetal programming from a placenta-specific mouse model. Autophagy 2016, 12:752-769

48. Barker DJ, Bull AR, Osmond C, Simmonds SJ: Fetal and placental size and risk of hypertension in adult life. BMJ 1990, 301:259-262

49. Bonds DR, Gabbe SG, Kumar S, Taylor T: Fetal weight/placental weight ratio and perinatal outcome. Am J Obstet Gynecol 1984, 149:195-200 